\title{
Backward extensions of hyperexpansive operators
}

\author{
by \\ Zenon J. JabŁoński (Kraków), Il Bong Jung (Daegu) \\ and JAN Stochel (Kraków)
}

\begin{abstract}
The concept of $k$-step full backward extension for subnormal operators is adapted to the context of completely hyperexpansive operators. The question of existence of $k$-step full backward extension is solved within this class of operators with the help of an operator version of the Levy-Khinchin formula. Some new phenomena in comparison with subnormal operators are found and related classes of operators are discussed as well.
\end{abstract}

1. Introduction. In 1976 A. Lambert, using a result of M. Embry [14], gave in [25] a characterization of subnormality for an abstract operator (bounded and linear) in terms of weighted shifts. It states that an injective operator $T$ acting on a (complex) Hilbert space $\mathcal{H}$ is subnormal if and only if for each nonzero vector $h$ in $\mathcal{H}$, the weighted shift $W_{T, h}$ with weight sequence $\left\{\left\|T^{n+1} h\right\| /\left\|T^{n} h\right\|\right\}_{n=0}^{\infty}$ is subnormal. In other words, the class of injective subnormal operators is an instance of an abstract class $\mathcal{C}$ of injective Hilbert space operators possessing the following property:

(P) an operator $T$ on $\mathcal{H}$ is of class $\mathcal{C}$ if and only if for every nonzero vector $h$ in $\mathcal{H}$, the weighted shift $W_{T, h}$ is of class $\mathcal{C}$.

It turns out that the class of injective paranormal operators $\left({ }^{1}\right)$ satisfies (P) as well. Indeed, $T$ is paranormal if and only if for each nonzero vector $h$ in $\mathcal{H}$, the sequence $\left\{\left\|T^{n+1} h\right\| /\left\|T^{n} h\right\|\right\}_{n=0}^{\infty}$ is weakly increasing (cf. [15, Addendum]) or equivalently the weighted shift $W_{T, h}$ is hyponormal. Since an injective weighted shift is hyponormal if and only if it is paranormal, our claim is proved. According to the above discussion, we see that the class

2000 Mathematics Subject Classification: Primary 47B20, 47B37; Secondary 44A60.

Key words and phrases: completely hyperexpansive operator, 2-isometry, unilateral weighted shift, the Levy-Khinchin formula, backward extension.

The work of the first and the third authors was supported by the KBN grant 2 P03A 037 024. The work of the second author was supported by a grant (R14-2003-006-01000-0) from the Korea Science and Engineering Research Foundation.

$\left({ }^{1}\right)$ Recall that $T$ is said to be paranormal if $\|T h\|^{2} \leq\|h\|\left\|T^{2} h\right\|$ for all $h \in \mathcal{H}$; notice also that hyponormal operators are paranormal (cf. $[18,15])$. 
of injective hyponormal operators does not have the property $(\mathrm{P})$ because there are injective paranormal operators which are not hyponormal (if $T$ is an injective hyponormal operator whose square is not hyponormal (cf. [19]), then by [15, Theorem 1], $T^{2}$ is the required paranormal operator; see also [15, Theorem 2] for the noninjective case).

Recently new classes of Hilbert space operators have attracted the attention of researchers. These are $m$-isometries [27, 2, 28, 3, 4, 5, 30, 23], $m$-hyperexpansive operators $[27,26,7,30,29,23]$ and completely hyperexpansive operators $[6,7,11,30,20,9,10,21,8,22]$. As shown in [23, Proposition 6.7], the classes of 2-isometries and 2-hyperexpansive operators have property $(\mathrm{P})$. It turns out that the classes of $m$-isometries, $m$-hyperexpansive operators and completely hyperexpansive operators share the same property (cf. Proposition 2.4 and Corollary 2.5).

In [12] R. Curto characterized subnormal weighted shifts which have backward extensions within the class of subnormal weighted shifts. Recall that a weighted shift with weights $\left\{\lambda_{n}\right\}_{n=0}^{\infty}$ has a subnormal (one-step) backward extension if for some positive scalar $\lambda_{-1}$, the weighted shift with weights $\left\{\lambda_{n-1}\right\}_{n=0}^{\infty}$ is subnormal. The concept of backward extension was studied in more detail in $[17,13]$ and extended to the case of abstract subnormal operators in [24].

In the present paper we adapt the concept of backward extension to completely hyperexpansive and 2-hyperexpansive operators (in fact, this can be done for other classes of operators mentioned above). The essential part of our paper deals with (injective and unilateral) weighted shifts. In Section 3 we discuss the question of existence of completely hyperexpansive backward extensions. The main result of this section, Theorem 3.2, provides a solution written in terms of measures associated with operators in question via the Levy-Khinchin formula. Moreover, the set of all measures associated with completely hyperexpansive backward extensions of the given operator is explicitly described.

Section 4 extends the main result of the previous one to the context of $k$-step backward extensions. Moreover, we show that a nonisometric completely hyperexpansive weighted shift has no completely hyperexpansive $\infty$ step backward extension (cf. Corollary 4.6). This is opposite to the case of nonisometric subnormal weighted shifts which allow for subnormal $\infty$-step backward extensions (cf. [24]).

In Section 5 we turn our attention to abstract completely hyperexpansive operators transplanting the notion of $k$-step full backward extension from subnormal operators to completely hyperexpansive ones. An operator version of the Levy-Khinchin formula plays a crucial role in this section. The next essential difference between subnormality and complete hyperexpansivity is emphasized by Proposition 5.8. Namely, it is proved that the prop- 
erty of having completely hyperexpansive $k$-step full backward extension is preserved by infinite orthogonal sums, which is not the case for subnormal operators (cf. [24]).

Section 6 deals with scalar and operator-valued weighted shifts. The third difference between subnormality and complete hyperexpansivity is exhibited in Example 6.5. It is shown there that the set of all completely hyperexpansive $k$-step backward extension vectors need not be a linear space (compare with [17, Corollary 5.4]). In the final section we discuss the question of existence of $k$-step full backward extension within the class of 2-hyperexpansive operators. This enables us to clarify the role played by 2 -isometries in our considerations.

2. Preliminaries. Let $\mathcal{H}$ be a (complex) Hilbert space. Denote by $\boldsymbol{B}(\mathcal{H})$ the $C^{*}$-algebra of all bounded linear operators on $\mathcal{H}$ and by $I_{\mathcal{H}}$ the identity operator on $\mathcal{H}$. From now on, all operators taken into consideration in this paper are assumed to be linear and bounded. Given $A, B \in \boldsymbol{B}(\mathcal{H})$, we write $A>B$ (resp. $A \geq B$ ) in case $\langle A h, h\rangle>\langle B h, h\rangle$ (resp. $\langle A h, h\rangle \geq\langle B h, h\rangle$ ) for every $h \in \mathcal{H} \backslash\{0\}$; we say that $A$ is positive if $A \geq 0$. As usual, $\ell^{2}$ stands for the Hilbert space of all square summable complex sequences and $\left\{e_{n}\right\}_{n=0}^{\infty}$ for the canonical orthonormal basis of $\ell^{2}$, i.e. $e_{n}=\left(\delta_{n, 0}, \delta_{n, 1}, \ldots\right)$, where $\delta_{k, l}$ is the Kronecker symbol. Denote by $\mathcal{B}([0,1])$ the $\sigma$-algebra of all Borel subsets of the closed unit segment $[0,1]$. For $x \in[0,1], \delta_{x}$ stands for the probability Borel measure on $[0,1]$ with total mass at $x$.

Given an operator $T \in \boldsymbol{B}(\mathcal{H})$ and an integer $n \geq 1$, we define the realvalued function $\varrho_{T, n}$ on $\mathcal{H}$ by

$$
\varrho_{T, n}(h):=\sum_{0 \leq p \leq n}(-1)^{p}\left(\begin{array}{l}
n \\
p
\end{array}\right)\left\|T^{p} h\right\|^{2}, \quad h \in \mathcal{H} .
$$

Definition 2.1 ([27, 7, 20,21]). Let $m \geq 1$ be an integer. We say that an operator $T \in \boldsymbol{B}(\mathcal{H})$ is

(a) $m$-isometric if $\varrho_{T, m}=0$,

(b) $m$-expansive if $\varrho_{T, m} \leq 0$,

(c) m-hyperexpansive if $\varrho_{T, n} \leq 0$ for all $n=1, \ldots, m$,

(d) completely hyperexpansive if $\varrho_{T, n} \leq 0$ for all $n \geq 1$.

The case $\varrho_{T, n} \geq 0$ is closely related to subnormal contractions (cf. [1]). The inclusion relations among the classes defined above are as follows:

2-isometry $\subseteq$ completely hyperexpansive $\subseteq \cdots \subseteq(m+1)$-hyperexpansive

$$
\subseteq m \text {-hyperexpansive } \subseteq \cdots \subseteq 1 \text {-hyperexpansive }
$$

Moreover, all these inclusions are proper (cf. [27] and [30]). 
An operator $W$ on $\ell^{2}$ of the form

$$
W\left(\xi_{0}, \xi_{1}, \ldots\right)=\left(0, \lambda_{0} \xi_{0}, \lambda_{1} \xi_{1}, \ldots\right), \quad\left(\xi_{0}, \xi_{1}, \ldots\right) \in \ell^{2},
$$

where $\left\{\lambda_{n}\right\}_{n=0}^{\infty}$ is a bounded sequence of positive real numbers, is called a (unilateral) weighted shift with weight sequence $\left\{\lambda_{n}\right\}_{n=0}^{\infty}$. Set $\beta_{n}(W)=$ $\left\|W^{n} e_{0}\right\|^{2}$ for $n \geq 0$, i.e. $\beta_{0}(W)=1$ and $\beta_{n}(W)=\lambda_{0}^{2} \cdots \lambda_{n-1}^{2}$ for $n \geq 1$. It is evident that the weights $\lambda_{n}$ of $W$ can be recovered from the sequence $\left\{\beta_{m}(W)\right\}_{m=0}^{\infty}$ as follows:

$$
\lambda_{n}=\sqrt{\frac{\beta_{n+1}(W)}{\beta_{n}(W)}}, \quad n \geq 0 .
$$

This means that the formula (2.1) establishes one-to-one correspondence between weighted shifts $W$ and sequences $\left\{\beta_{m}(W)\right\}_{m=0}^{\infty}$. In case $\lambda_{n}=1$ for all $n \geq 0, W$ is called the isometric unilateral shift.

The following result characterizes completely hyperexpansive weighted shifts (see [7, Proposition 3], [20, Lemma 4.1] and [23, Lemma 6.1]).

Proposition 2.2. A weighted shift $W$ with weight sequence $\left\{\lambda_{n}\right\}_{n=0}^{\infty}$ is completely hyperexpansive if and only if there exists a finite positive Borel measure $\mu$ on $[0,1]$ such that

$$
\beta_{n}(W)=1+\int_{[0,1]}\left(1+x+\cdots+x^{n-1}\right) d \mu(x), \quad n \geq 1 .
$$

The correspondence $W \leftrightarrow \mu$ is one-to-one (in particular, the measure $\mu$ in (2.2) is unique). Moreover, the weighted shift $W$ is a 2-isometry if and only if the corresponding measure $\mu$ is of the form $\alpha \delta_{1}$, where $\alpha$ is a nonnegative real number.

Definition 2.3. If (2.2) holds, then we say that the measure $\mu$ is associated with the weighted shift $W$ or that $W$ is associated with $\mu$.

Let us recall that if $T \in \boldsymbol{B}(\mathcal{H})$ is injective and $h \in \mathcal{H} \backslash\{0\}$, then $W_{T, h}$ stands for the weighted shift with weight sequence $\left\{\left\|T^{n+1} h\right\| /\left\|T^{n} h\right\|\right\}_{n=0}^{\infty}$ (the operator $W_{T, h}$ is bounded because the sequence of its weights is bounded above by $\|T\|$ ). Now we show that the classes of (hyper-) expansive operators defined above have the property $(\mathrm{P})$ mentioned in Section 1 (for the case of 2-hyperexpansive and 2-isometric operators see [23, Proposition 6.7]).

Proposition 2.4. If $T \in \boldsymbol{B}(\mathcal{H})$ and $m \geq 1$ is an integer, then the following conditions are equivalent:

(i) $T$ is $m$-isometric (resp. m-expansive, m-hyperexpansive),

(ii) $T$ is injective and $W_{T, h}$ is $m$-isometric (resp. m-expansive, m-hyperexpansive) for every nonzero vector $h \in \mathcal{H}$. 
Proof. If $T$ is $m$-expansive and $T h=0$ for some $h \in \mathcal{H}$, then

$$
\|h\|^{2}=\varrho_{T, m}(h) \leq 0,
$$

which yields the injectivity of $T$. It is easily seen that a weighted shift $W$ is $m$-isometric (resp. $m$-expansive) if and only if $\varrho_{W, m}\left(e_{n}\right)=0$ (resp. $\varrho_{W, m}\left(e_{n}\right)$ $\leq 0)$ for every integer $n \geq 0$. This and the equality

$$
\varrho_{W_{T, h}, m}\left(e_{n}\right)=\frac{\varrho_{T, m}\left(T^{n} h\right)}{\left\|T^{n} h\right\|^{2}}, \quad h \in \mathcal{H} \backslash\{0\}, n \geq 0,
$$

complete the proof.

Corollary 2.5. An operator $T \in \boldsymbol{B}(\mathcal{H})$ is completely hyperexpansive if and only if $T$ is injective and the weighted shift $W_{T, h}$ is completely hyperexpansive for every $h \in \mathcal{H} \backslash\{0\}$. If this is the case, then for every $h \in \mathcal{H} \backslash\{0\}$, there exists a unique finite positive Borel measure $\mu_{T, h}$ on $[0,1]$ such that

$$
\beta_{n}\left(W_{T, h}\right)=\frac{\left\|T^{n} h\right\|^{2}}{\|h\|^{2}}=1+\int_{[0,1]}\left(1+x+\cdots+x^{n-1}\right) d \mu_{T, h}(x), \quad n \geq 1 .
$$

\section{Completely hyperexpansive backward extensions}

Definition 3.1. We say that a weighted shift $W$ with weight sequence $\left\{\lambda_{n}\right\}_{n=0}^{\infty}$ has a completely hyperexpansive (one-step) backward extension if for some positive scalar $\lambda_{-1}$, the weighted shift $W^{\prime}$ with weight sequence $\left\{\lambda_{n-1}\right\}_{n=0}^{\infty}$ is completely hyperexpansive; the weighted shift $W^{\prime}$ will be called a completely hyperexpansive backward extension of $W$.

Regarding Definition 3.1, we see that the operator $W$ may be identified (up to unitary equivalence) with the restriction $W^{\prime} \mid \bigvee_{n=1}^{\infty}\left\{e_{n}\right\}$ of $W^{\prime}$ to $\bigvee_{n=1}^{\infty}\left\{e_{n}\right\}$, the closed linear span of $\left\{e_{n}\right\}_{n=1}^{\infty}$. This means that $W$ itself is completely hyperexpansive.

The following result characterizes those weighted shifts which have completely hyperexpansive backward extensions.

THEOREM 3.2. Let $W$ be a completely hyperexpansive weighted shift with weight sequence $\left\{\lambda_{n}\right\}_{n=0}^{\infty}$ and with associated measure $\mu$. Then $W$ has a completely hyperexpansive backward extension if and only if $\left({ }^{2}\right)$

$$
\int_{[0,1]} \frac{1}{x} d \mu(x)<1
$$

If this is the case, then the measures $\nu$ associated with completely hyperexpansive backward extensions of $W$ are in one-to-one correspondence with the

$\left({ }^{2}\right)$ In the formula (3.1), we adhere to the convention that $\frac{1}{0}=\infty$. In particular, (3.1) implies $\mu(\{0\})=0$. 
nonnegative real numbers $t$ via

$$
\nu(\sigma)=\frac{1+t}{1-\int_{[0,1]} \frac{1}{x} d \mu(x)} \int_{\sigma} \frac{1}{x} d \mu(x)+t \delta_{0}(\sigma), \quad \sigma \in \mathcal{B}([0,1]) .
$$

Proof. Let $W^{\prime}$ be a completely hyperexpansive backward extension of $W$ and let $\nu$ be a measure associated with $W^{\prime}$. It is easily seen that (cf. Definition 3.1)

$$
\beta_{n+1}\left(W^{\prime}\right)=\lambda_{-1}^{2} \beta_{n}(W), \quad n \geq 0 .
$$

This and Definition 2.3 imply

$$
\begin{aligned}
\int_{[0,1]} x^{n} x d \nu(x) & =\beta_{n+2}\left(W^{\prime}\right)-\beta_{n+1}\left(W^{\prime}\right)=\lambda_{-1}^{2}\left(\beta_{n+1}(W)-\beta_{n}(W)\right) \\
& =\int_{[0,1]} x^{n} \lambda_{-1}^{2} d \mu(x), \quad n \geq 0
\end{aligned}
$$

which, by the uniqueness of the solution of the Hausdorff moment problem, yields

$$
\lambda_{-1}^{2} \mu(\sigma)=\int_{\sigma} x d \nu(x), \quad \sigma \in \mathcal{B}([0,1]) .
$$

In consequence, we have $\mu(\{0\})=0$ and

$$
\nu(\sigma)=\lambda_{-1}^{2} \int_{\sigma} \frac{1}{x} d \mu(x)+\nu(\{0\}) \delta_{0}(\sigma), \quad \sigma \in \mathcal{B}([0,1]) .
$$

Applying (2.2) (to $W^{\prime}$ and $\nu$ in place of $W$ and $\mu$ ) and (3.4), we get

$$
\lambda_{-1}^{2}=\beta_{1}\left(W^{\prime}\right)=1+\nu([0,1])=1+\nu(\{0\})+\lambda_{-1}^{2} \int_{[0,1]} \frac{1}{x} d \mu(x) .
$$

This leads to

$$
\lambda_{-1}^{2}\left(1-\int_{[0,1]} \frac{1}{x} d \mu(x)\right)=1+\nu(\{0\}) .
$$

From (3.5) and $\lambda_{-1}>0$ we infer that (3.1) holds. Computing $\lambda_{-1}^{2}$ from (3.5) and then substituting it into (3.4) we come to (3.2) with $t=\nu(\{0\})$.

Suppose now that (3.1) holds. Let $W^{\prime}$ be the weighted shift with associated measure $\nu$ given by (3.2). Then we have

$$
\beta_{1}\left(W^{\prime}\right)=1+\nu([0,1])=\frac{1+t}{1-\int_{[0,1]} \frac{1}{x} d \mu(x)} .
$$

One can deduce from Definition 2.3, (3.2) and (3.6) that for every $n \geq 1$,

$$
\beta_{n+1}\left(W^{\prime}\right)=1+\nu([0,1])+\beta_{1}\left(W^{\prime}\right) \int_{[0,1]} \frac{x+\cdots+x^{n}}{x} d \mu(x)=\beta_{1}\left(W^{\prime}\right) \beta_{n}(W),
$$


which implies (3.3) with $\lambda_{-1}:=\sqrt{\beta_{1}\left(W^{\prime}\right)}$. This and (2.1) show that $W^{\prime}$ is a completely hyperexpansive backward extension of $W$.

That the correspondence $t \leftrightarrow W^{\prime}$ is one-to-one follows from Proposition 2.2 and the equality $\nu(\{0\})=t$, which in turn is a consequence of $(3.2)$. This completes the proof.

The following corollary may be thought of as the complete hyperexpansive counterpart of [12, Proposition 8].

COROLlary 3.3. Let $W$ be a weighted shift with weight sequence $\left\{\lambda_{n}\right\}_{n=0}^{\infty}$. Suppose that the restriction of $W$ to $\bigvee_{n=1}^{\infty}\left\{e_{n}\right\}$ is completely hyperexpansive $\left({ }^{3}\right)$ with associated measure $\mu$. Then $W$ is completely hyperexpansive if and only if the following two conditions hold:

(i) $\int_{[0,1]} \frac{1}{x} d \mu(x)<1$,

(ii) $\lambda_{0}^{2} \geq\left(1-\int_{[0,1]} \frac{1}{x} d \mu(x)\right)^{-1}$.

Proof. Applying Theorem 3.2 and (3.6), we see that $W$ is completely hyperexpansive $\left({ }^{4}\right)$ if and only if (i) is valid and

$$
\lambda_{0}^{2}=\beta_{1}(W)=\frac{1+t}{1-\int_{[0,1]} \frac{1}{x} d \mu(x)} \quad \text { for some } t \geq 0 .
$$

Since (3.7) is equivalent to (ii), the proof is complete.

COROLlaRY 3.4. The restriction of any completely hyperexpansive weighted shift to $\bigvee_{n=1}^{\infty}\left\{e_{n}\right\}$ has infinitely many completely hyperexpansive backward extensions.

\section{Completely hyperexpansive $k$-step backward extensions}

Definition 4.1. Given an integer $k \geq 1$, we say that a weighted shift $W$ with weight sequence $\left\{\lambda_{n}\right\}_{n=0}^{\infty}$ has a completely hyperexpansive $k$-step backward extension if for some positive scalars $\lambda_{-k}, \ldots, \lambda_{-1}$, the weighted shift $V$ with weight sequence $\left\{\lambda_{n-k}\right\}_{n=0}^{\infty}$ is completely hyperexpansive; the weighted shift $V$ will be called a completely hyperexpansive $k$-step backward extension of $W$.

Regarding Definition 4.1, we see that the operator $W$ may be identified (up to unitary equivalence) with $\left.V\right|_{\bigvee_{n=k}^{\infty}} ^{\infty}\left\{e_{n}\right\}$. Hence $W$ itself is completely hyperexpansive. It is a matter of routine to show that a weighted shift $W$ has a completely hyperexpansive $k$-step backward extension if and only if it has a

$\left({ }^{3}\right)$ This is the same as saying that the weighted shift with weight sequence $\left\{\lambda_{n+1}\right\}_{n=0}^{\infty}$ is completely hyperexpansive.

$\left({ }^{4}\right)$ Note that $W$ can be thought of as a completely hyperexpansive backward extension of $\left.W\right|_{\bigvee_{n=1}^{\infty}}\left\{e_{n}\right\}$. 
completely hyperexpansive $(k-1)$-step backward extension $V^{\prime}$ which in turn has a completely hyperexpansive (one-step) backward extension $(k \geq 2)$.

THEOREM 4.2. Let $k \geq 1$ be an integer and $W$ be a completely hyperexpansive weighted shift with weight sequence $\left\{\lambda_{n}\right\}_{n=0}^{\infty}$ and with associated measure $\mu$. Then

$1^{\circ} W$ has a completely hyperexpansive $k$-step backward extension if and only if

$$
\int_{[0,1]}\left(\frac{1}{x}+\cdots+\frac{1}{x^{k}}\right) d \mu(x)<1,
$$

$2^{\circ}$ if (4.1) holds, then the measures $\zeta$ associated with completely hyperexpansive $k$-step backward extensions of $W$ are in one-to-one correspondence with the nonnegative real numbers $t$ via

$$
\begin{aligned}
\zeta(\sigma)=\frac{1+t}{1-\int_{[0,1]}\left(\frac{1}{x}+\cdots+\frac{1}{x^{k}}\right) d \mu(x)} \int_{\sigma} \frac{1}{x^{k}} d \mu(x)+t \delta_{0}(\sigma), & \\
& \sigma \in \mathcal{B}([0,1]),
\end{aligned}
$$

$3^{\circ}$ if (4.1) holds, then there exists at most one completely hyperexpansive $k$-step backward extension $V$ of $W$ which has a completely hyperexpansive backward extension; the weighted shift $V$ is associated with the measure $\zeta$ given by (4.2) with $t=0$.

Proof. $3^{\circ}$ can be inferred from $2^{\circ}$ via Theorem 3.2 (see also footnote $\left({ }^{2}\right)$ ). To prove $1^{\circ}$ and $2^{\circ}$, we proceed by induction on $k$. The case $k=1$ is covered by Theorem 3.2. We show how to pass from $k$ to $k+1$. According to Theorem 3.2, the weighted shift with associated measure $\zeta$ given by (4.2) has a completely hyperexpansive backward extension if and only if $t=\zeta(\{0\})=0$ and

$$
\frac{1}{1-\int_{[0,1]}\left(\frac{1}{x}+\cdots+\frac{1}{x^{k}}\right) d \mu(x)} \int_{[0,1]} \frac{1}{x^{k+1}} d \mu(x)=\int_{[0,1]} \frac{1}{x} d \zeta(x)<1,
$$

which in turn is equivalent to the conjunction of $t=0$ and

$$
\int_{[0,1]}\left(\frac{1}{x}+\cdots+\frac{1}{x^{k+1}}\right) d \mu(x)<1 .
$$

This implies $1^{\circ}$ with $k+1$ in place of $k$. If (4.4) holds, then in view of $3^{\circ}$, (4.3) and Theorem 3.2 there exists exactly one completely hyperexpansive $k$-step backward extension $V$ of $W$ which has a completely hyperexpansive backward extension; the weighted shift $V$ is associated with the measure $\zeta$ given by (4.2) with $t=0$. Applying Theorem 3.2 to this particular measure and employing (4.3), we conclude that all measures $\xi$ associated with 
completely hyperexpansive $(k+1)$-step backward extensions of $W$ are of the form

$$
\begin{aligned}
\xi(\sigma) & =\frac{1+t}{1-\int_{[0,1]} \frac{1}{x} d \zeta(x)} \int_{\sigma} \frac{1}{x} d \zeta(x)+t \delta_{0}(\sigma) \\
= & \frac{(1+t) \int_{\sigma} \frac{1}{x^{k+1}} d \mu(x)}{\left(1-\frac{\int_{[0,1]} \frac{1}{x^{k+1}} d \mu(x)}{1-\int_{[0,1]}\left(\frac{1}{x}+\cdots+\frac{1}{x^{k}}\right) d \mu(x)}\right)\left(1-\int_{[0,1]}\left(\frac{1}{x}+\cdots+\frac{1}{x^{k}}\right) d \mu(x)\right)} \\
& +t \delta_{0}(\sigma) \\
= & \frac{1+t}{1-\int_{[0,1]}\left(\frac{1}{x}+\cdots+\frac{1}{x^{k+1}}\right) d \mu(x)} \int_{\sigma} \frac{1}{x^{k+1}} d \mu(x)+t \delta_{0}(\sigma), \quad \sigma \in \mathcal{B}([0,1]),
\end{aligned}
$$

where $t$ runs over the segment $[0, \infty)$. This completes the proof.

REMARK 4.3. The reader can verify that the probability measures $\zeta$ associated with subnormal $k$-step backward extensions of a fixed subnormal weighted shift $W$ are in one-to-one correspondence with the real numbers $t \in[0,1)$ via

$$
\zeta(\sigma)=\frac{1-t}{\int_{[0, \infty)} \frac{1}{x^{k}} d \mu(x)} \int_{\sigma} \frac{1}{x^{k}} d \mu(x)+t \delta_{0}(\sigma)
$$

$$
\text { for any Borel subset } \sigma \text { of }[0, \infty) \text {, }
$$

where $\mu$ is the Stieltjes moment measure associated with the weighted shift $W$ (for definitions and related results, see [12]).

Corollary 4.4 below is related to Corollary 3.5 of [17]. It deals with finite perturbations of weights of completely hyperexpansive weighted shifts, while its counterpart in [17] with one-point perturbations of weights of subnormal weighted shifts.

Corollary 4.4. Let $W_{1}$ and $W_{2}$ be completely hyperexpansive weighted shifts with weight sequences $\left\{\lambda_{n}^{(1)}\right\}_{n=0}^{\infty}$ and $\left\{\lambda_{n}^{(2)}\right\}_{n=0}^{\infty}$, respectively. If there exists $N \geq 1$ such that $\lambda_{n}^{(1)}=\lambda_{n}^{(2)}$ for all $n \geq N$, then $\lambda_{n}^{(1)}=\lambda_{n}^{(2)}$ for all $n \geq 1$.

Proof. It suffices to consider the case $N \geq 2$. Since the weighted shifts $V_{1}$ and $V_{2}$ with weight sequences $\left\{\lambda_{n+1}^{(1)}\right\}_{n=0}^{\infty}$ and $\left\{\lambda_{n+1}^{(2)}\right\}_{n=0}^{\infty}$, respectively, are completely hyperexpansive $(N-1)$-step backward extensions of the weighted shift with weight sequence $\left\{\lambda_{n+N}^{(1)}\right\}_{n=0}^{\infty}$, and both have completely hyperexpansive backward extensions (namely $W_{1}$ and $W_{2}$, respectively), we infer from parts $1^{\circ}$ and $3^{\circ}$ of Theorem 4.2 (with $k=N-1$ ) that $V_{1}=V_{2}$, which completes the proof. 
REMARK 4.5. Corollary 4.4 can be rephrased as follows: changing finitely many weights of a weighted shift including at least one of its weights with index $s \geq 1$ does destroy complete hyperexpansivity. Corollary 3.4 explains why the weighted shifts $W_{1}$ and $W_{2}$ satisfying the assumptions of Corollary 4.4 need not coincide in general. It has been proved in [23, Proposition 6.2 (vii)] that if two successive weights $\lambda_{s}$ and $\lambda_{s+1}$ of a 2-hyperexpansive weighted shift $W$ coincide, then $\lambda_{n}=1$ for all $n \geq s$. If additionally $W$ is completely hyperexpansive, we can show more, namely that all the weights of $W$, except possibly $\lambda_{0}$, must be equal to 1 (apply Corollary 4.4 to $W_{1}=W$ and $W_{2}=$ the isometric unilateral shift).

The following corollary shows that a completely hyperexpansive weighted shift which is not isometric has no completely hyperexpansive $\infty$-step backward extension. This is a new phenomenon compared with nonisometric subnormal weighted shifts which allow for subnormal $\infty$-step backward extensions (cf. [24, Example 4.5]).

For a weighted shift $W$, we define the quantity $\varkappa_{W}$ as follows: if $W$ does not have any completely hyperexpansive backward extension, we set $\varkappa_{W}=0$; if such an extension exists, $\varkappa_{W}$ stands for the maximum integer $k \geq 1$ for which $W$ has a completely hyperexpansive $k$-step backward extension provided the maximum exists; otherwise we set $\varkappa_{W}=\infty$. We say that a sequence $\left\{a_{n}\right\}_{n=1}^{\infty} \subseteq \mathbb{R} \cup\{\infty\}$ is strictly increasing within $\mathbb{R}$ if either $\left\{a_{n}\right\}_{n=1}^{\infty} \subseteq \mathbb{R}$ and $\left\{a_{n}\right\}_{n=1}^{\infty}$ is strictly increasing, or there exists an integer $m \geq 1$ such that $\left\{a_{n}\right\}_{n=1}^{m} \subseteq \mathbb{R},\left\{a_{n}\right\}_{n=1}^{m}$ is strictly increasing and $a_{n}=\infty$ for every $n \geq m+1$.

COROLlary 4.6. Let $W$ be a completely hyperexpansive weighted shift with associated measure $\mu$. Set

$$
\tau_{k}=\int_{[0,1]}\left(\frac{1}{x}+\cdots+\frac{1}{x^{k}}\right) d \mu(x) \quad \text { for } k \geq 1 .
$$

Then

(i) $\varkappa_{W}=\infty$ if and only if $W$ is the isometric unilateral shift $\left({ }^{5}\right)$,

(ii) if $1 \leq \varkappa_{W}<\infty$, then the sequence $\left\{\tau_{k}\right\}_{k=1}^{\infty}$ is strictly increasing within $\mathbb{R}, \lim _{k \rightarrow \infty} \tau_{k}=\infty$ and $\varkappa_{W}=\max \left\{k \geq 1: \tau_{k}<1\right\}$,

(iii) if $1 \leq \varkappa_{W}<\infty$, then $\beta_{1}(W)>1$ and $\varkappa_{W}<1 /\left(\beta_{1}(W)-1\right)$.

Proof. (i) It suffices to prove the "only if" part of (i). Theorem 4.2 yields

$$
k \mu([0,1]) \leq \tau_{k}<1, \quad k \geq 1,
$$

which implies $\mu=0$. This, Proposition 2.2 and (2.1) complete the proof of (i).

$\left({ }^{5}\right)$ Assertion (i) is a particular case of Proposition 7.1. 
(ii) Suppose that $1 \leq \varkappa_{W}<\infty$. Then clearly $\mu \neq 0$ and, by Theorem 4.2, $\mu(\{0\})=0$. Hence $\int_{[0,1]} \frac{1}{x^{k+1}} d \mu(x)>0$ for every integer $k \geq 0$. This, the inequality $k \mu([0,1]) \leq \tau_{k}(k \geq 1)$ and Theorem 4.2 imply (ii).

(iii) This follows from (4.5) applied to $k=\varkappa_{W}$ and (2.2).

EXAMPLE 4.7. Let us discuss some examples of completely hyperexpansive weighted shifts. In view of $(2.1)$ and Proposition 2.2 , the completely hyperexpansive weighted shift $W^{(\alpha)}$ with associated measure $\mu=\alpha \delta_{1}$, where $\alpha$ is a nonnegative real number, is a 2 -isometry with weight sequence

$$
\left\{\sqrt{\frac{1+(n+1) \alpha}{1+n \alpha}}\right\}_{n=0}^{\infty},
$$

and all 2-isometric weighted shifts are of this form. There are two cases which are of particular interest, namely $\alpha=0$ and $\alpha=1$, which correspond to the isometric unilateral shift and the Dirichlet shift, respectively. By Theorem 4.2 , for every $k \geq 1, W^{(\alpha)}$ has a completely hyperexpansive $k$-step backward extension if and only if $k \alpha<1$. This automatically excludes the Dirichlet shift from the class of weighted shifts which have completely hyperexpansive backward extensions. In our considerations the Dirichlet shift plays a similar role to the Bergman shift in the theory of subnormal backward extensions; namely, the Bergman shift (which is the weighted shift with weights $\left.\{\sqrt{(n+1) /(n+2)}\}_{n=0}^{\infty}\right)$ does not have any subnormal backward extension (cf. [17, Example 4.2]). Reverting to the case of arbitrary $\alpha$, note that if $W^{(\alpha)}$ has a completely hyperexpansive $k$-step backward extension (i.e. $k \alpha<1$ ), then by Theorem 4.2 all measures $\zeta$ associated with completely hyperexpansive $k$-step backward extensions of $W^{(\alpha)}$ are of the form

$$
\zeta=\frac{(1+t) \alpha}{1-k \alpha} \delta_{1}+t \delta_{0}, \quad t \geq 0 .
$$

The completely hyperexpansive weighted shift with associated measure $\zeta$ is 2 -isometric if and only if $t=0$. This means that $W^{(\alpha)}$ has a lot of completely hyperexpansive $k$-step backward extensions, but exactly one of them is 2isometric.

We conclude Example 4.7 with a somewhat surprising observation. Namely, according to the above discussion, a 2-isometric weighted shift $W$ has a completely hyperexpansive backward extension if and only if $\beta_{1}(W)<2$ (because $\alpha=\beta_{1}(W)-1$ ). On the other hand, the inequality $\beta_{1}(W)<2$ does not guarantee that an arbitrary completely hyperexpansive weighted shift $W$ has a completely hyperexpansive backward extension. Indeed, considering $\mu=\alpha \delta_{0}$ with $\alpha \in(0,1)$ we see that $\beta_{1}(W)=1+\alpha<2$ and $W$ has no completely hyperexpansive backward extension. 
5. Completely hyperexpansive $k$-step full backward extensions. Let $T \in \boldsymbol{B}(\mathcal{H})$ be a completely hyperexpansive operator. In view of Corollary 2.5, for every $h \in \mathcal{H} \backslash\{0\}$, the weighted shift $W_{T, h}$ is completely hyperexpansive. Denote by $\mu_{T, h}$ the measure associated with $W_{T, h}$. By Theorem 4.2, $W_{T, h}$ has a completely hyperexpansive $k$-step backward extension if and only if

$$
\int_{[0,1]} \varphi_{k}(x) d \mu_{T, h}(x)<1,
$$

where

$$
\varphi_{k}(x)= \begin{cases}\frac{1}{x}+\cdots+\frac{1}{x^{k}} & \text { for } x \in(0,1], \quad k \geq 1 . \\ \infty & \text { for } x=0,\end{cases}
$$

Definition 5.1. Let $k \geq 1$ be an integer and $T \in \boldsymbol{B}(\mathcal{H})$ be a completely hyperexpansive operator. A nonzero vector $h \in \mathcal{H}$ is called a completely hyperexpansive $k$-step backward extension vector for $T$ if $W_{T, h}$ has a completely hyperexpansive $k$-step backward extension; the set of all such vectors together with the zero vector is denoted by $\mathcal{E}_{T, k}$. If $\mathcal{E}_{T, k}=\mathcal{H}$, then we say that $T$ has completely hyperexpansive $k$-step full backward extension.

The set $\mathcal{E}_{T, k}$ need not be a linear subspace of $\mathcal{H}$ (cf. Example 6.5). We now show that the restriction $T_{k}$ of a completely hyperexpansive operator $T$ to the range of $T^{k}$ has completely hyperexpansive $k$-step full backward extension, and that this "virtual" extension is closely related to the "real" one $T$ (see also Example 6.6 which illustrates Proposition 5.2 and Corollary $5.3)$.

Proposition 5.2. If $k \geq 1$ is an integer and $T \in \boldsymbol{B}(\mathcal{H})$ is a completely hyperexpansive operator, then the following two conditions hold:

(i) the space $T^{k}(\mathcal{H})$ is closed, the operator $T_{k}:=\left.T\right|_{T^{k}(\mathcal{H})}$ is completely hyperexpansive and $T^{k}(\mathcal{H})=\mathcal{E}_{T_{k}, k} \subseteq \mathcal{E}_{T, k} ;$ moreover, for every nonzero vector $h$ in $T^{k}(\mathcal{H})$, the weighted shift $W_{T, T^{-k} h}$ is a completely hyperexpansive $k$-step backward extension of $W_{T_{k}, h}\left(=W_{T, h}\right)$ and

$$
\begin{aligned}
& \mu_{T, T^{-k} h}(\sigma)=\frac{1+t_{h}}{1-\int_{[0,1]} \varphi_{k} d \mu_{T, h}} \int_{\sigma} \frac{1}{x^{k}} d \mu_{T, h}(x)+t_{h} \delta_{0}(\sigma), \\
& \qquad \sigma \in \mathcal{B}([0,1]),
\end{aligned}
$$

where

$$
t_{h}=\frac{\left\|T^{-(k-1)} h\right\|^{2}\left(1-\int_{[0,1]} \varphi_{k} d \mu_{T, h}\right)}{\left\|T^{-k} h\right\|^{2}\left(1-\int_{[0,1]} \varphi_{k-1} d \mu_{T, h}\right)}-1, \quad \varphi_{0}:=0,
$$

(ii) if $\mu_{T, g}(\{0\})=0$ for every $g \in \mathcal{H}$, then $t_{h}=0$ for all $h \in T^{k}(\mathcal{H}) \backslash\{0\}$. 
Proof. (i) By [27, Lemma 1], the operator $T$ is injective and the space $T(\mathcal{H})$ is closed. This in turn implies that $T^{k}(\mathcal{H})$ is closed as well (see [24, Remark 2.7]). It follows directly from Definition 2.1 that $T_{k}$ is completely hyperexpansive. If $h \in T^{k}(\mathcal{H})$ is nonzero, then in view of Corollary 2.5 the weighted shift $W_{T, T^{-k} h}$ is a completely hyperexpansive $k$-step backward extension of $W_{T_{k}, h}$. Hence by part $2^{\circ}$ of Theorem 4.2 the measure $\mu_{T, T^{-k} h}$ is of the form (5.2) with a unique $t_{h} \geq 0$. Applying (2.2) and (5.2), we get

$$
\begin{aligned}
\beta_{1}\left(W_{T, T^{-k} h}\right) & =1+\mu_{T, T^{-k} h}([0,1]) \\
& =1+\frac{1+t_{h}}{1-\int_{[0,1]} \varphi_{k} d \mu_{T, h}} \int_{[0,1]} \frac{1}{x^{k}} d \mu_{T, h}(x)+t_{h} .
\end{aligned}
$$

Computing $t_{h}$ from (5.4) leads to (5.3).

(ii) By (5.2), we have $t_{h}=\mu_{T, T^{-k} h}(\{0\})=0$ for every $h \in T^{k}(\mathcal{H}) \backslash\{0\}$, which completes the proof.

Corollary 5.3. Let $k, r \geq 1$ be integers and $T \in \boldsymbol{B}(\mathcal{H})$ be a completely hyperexpansive operator such that $\mu_{T, g}(\{0\})=0$ for every $g \in \mathcal{H}$. If $T_{k}:=$ $\left.T\right|_{T^{k}(\mathcal{H})}$ has completely hyperexpansive $(k+r)$-step full backward extension, then $T$ has completely hyperexpansive r-step full backward extension.

Proof. Take a nonzero vector $g \in \mathcal{H}$ and set $h:=T^{k}(g)$. By our assumption, the weighted shift $W_{T_{k}, h}$ has a completely hyperexpansive $(k+r)$ step backward extension, say $V$. In turn, according to Proposition 5.2, the weighted shift $W_{T, g}=W_{T, T^{-k} h}$ is a completely hyperexpansive $k$-step backward extension of $W_{T_{k}, h}$ and (5.2) holds with $t_{h}=0$. By Theorem 4.2, $W_{T, g}$ can be identified (up to unitary equivalence) with $\left.V\right|_{\bigvee_{n=r}^{\infty}\left\{e_{n}\right\}}$. Hence, $V$ is a completely hyperexpansive $r$-step backward extension of $W_{T, g}$, which completes the proof.

Let us recall an operator version of the Levy-Khinchin formula for completely hyperexpansive operators.

THEOREM 5.4. An operator $T \in \boldsymbol{B}(\mathcal{H})$ is completely hyperexpansive if and only if there exists a (unique) positive-operator-valued Borel measure $\left({ }^{6}\right)$ $F_{T}$ on $[0,1]$ such that

$$
T^{* n} T^{n}=I_{\mathcal{H}}+\int_{[0,1]}\left(1+x+\cdots+x^{n-1}\right) F_{T}(d x), \quad n \geq 1 .
$$

If this is the case, then

$$
\mu_{T, h}(\sigma)=\frac{1}{\|h\|^{2}}\left\langle F_{T}(\sigma) h, h\right\rangle, \quad \sigma \in \mathcal{B}([0,1]), h \in \mathcal{H} \backslash\{0\} .
$$

$\left({ }^{6}\right) F_{T}$ is $\sigma$-additive with respect to the weak operator topology; we do not assume that $F_{T}([0,1])=I_{\mathcal{H}}$. 
Proof. The first part of the conclusion follows from [20, Theorem 4.2]. Then (5.6) is a consequence of (5.5) and Corollary 2.5.

Definition 5.5. If (5.5) holds, then we say that the operator measure $F_{T}$ is associated with the operator $T$ or that $T$ is associated with $F_{T}$.

Following [31, Appendix], we write $\psi \in L^{1}\left(F_{T}\right)$ in case $\psi$ is a complex Borel function on $[0,1]$ and $\int_{[0,1]}|\psi(x)|\left\langle F_{T}(d x) h, h\right\rangle<\infty$ for all $h \in \mathcal{H}$. If this is the case, then there exists a unique operator $\int_{[0,1]} \psi d F_{T} \in \boldsymbol{B}(\mathcal{H})$ such that

$$
\left\langle\int_{[0,1]} \psi d F_{T} h, h\right\rangle=\int_{[0,1]} \psi(x)\left\langle F_{T}(d x) h, h\right\rangle, \quad h \in \mathcal{H} .
$$

Moreover,

$$
\left\|\int_{[0,1]} \psi d F_{T}\right\| \leq\left\|\int_{[0,1]}|\psi| d F_{T}\right\|=\sup _{\|h\|=1} \int_{[0,1]}|\psi(x)|\left\langle F_{T}(d x) h, h\right\rangle .
$$

If $\psi$ is nonnegative, then $\int_{[0,1]} \psi d F_{T} \geq 0$.

TheOREm 5.6. Let $T \in \boldsymbol{B}(\mathcal{H})$ be a completely hyperexpansive operator with associated operator measure $F_{T}$ and let $k \geq 1$ be an integer. Then the following conditions are equivalent:

(i) T has completely hyperexpansive $k$-step full backward extension,

(ii) $\varphi_{k} \in L^{1}\left(F_{T}\right)$ and $\int_{[0,1]} \varphi_{k} d F_{T}<I_{\mathcal{H}}$,

(iii) $\varphi_{k} \in L^{1}\left(F_{T}\right),\left\|\int_{[0,1]} \varphi_{k} d F_{T}\right\| \leq 1$ and 1 is not an eigenvalue of the operator $\int_{[0,1]} \varphi_{k} d F_{T}$.

Moreover, if $T$ has completely hyperexpansive $k$-step full backward extension, then

$$
\begin{aligned}
& F_{T}([0,1])<\frac{1}{k} I_{\mathcal{H}}, \\
& T^{*} T<\frac{k+1}{k} I_{\mathcal{H}} .
\end{aligned}
$$

Proof. The equivalence (i) $\Leftrightarrow($ ii) can be deduced from the equality (5.7) and Theorems 4.2 and 5.4. The equivalence (ii) $\Leftrightarrow$ (iii) is a consequence of the following fact: if $A \in \boldsymbol{B}(\mathcal{H})$ is a positive contraction, then

$$
\begin{aligned}
\langle A h, h\rangle=\langle h, h\rangle & \Leftrightarrow\left\|\left(I_{\mathcal{H}}-A\right)^{1 / 2} h\right\|^{2}=0 \\
& \Leftrightarrow\left(I_{\mathcal{H}}-A\right) h=0, \quad h \in \mathcal{H} .
\end{aligned}
$$

If (ii) holds, then

$$
k F_{T}([0,1]) \leq \int_{[0,1]} \varphi_{k} d F_{T}<I_{\mathcal{H}}
$$

which implies (5.9). Finally, (5.10) follows from (5.9) and (5.5) applied to $n=1$. 
The inequality (5.10) (and consequently (5.9)) can also be inferred from Corollary 7.2. It becomes optimal in the case of 2 -isometric operators.

Corollary 5.7. A 2-isometric operator $T \in \boldsymbol{B}(\mathcal{H})$ has completely hyperexpansive $k$-step full backward extension if and only if (5.10) holds.

Proof. By Proposition 4.5 of [20], there exists a positive operator $C \in$ $\boldsymbol{B}(\mathcal{H})$ such that $F_{T}(\sigma)=\delta_{1}(\sigma) C$ for $\sigma \in \mathcal{B}([0,1])$. Applying Theorem 5.6 completes the proof.

The following proposition emphasizes the essential difference between subnormal and completely hyperexpansive operators regarding backward extensions of orthogonal sums. In contradistinction to subnormal operators, the property of having completely hyperexpansive $k$-step full backward extension is preserved by the operation of taking infinite orthogonal sums (cf. Proposition 2.9 and Example 2.10 in [24]).

Proposition 5.8. Let $T=\bigoplus_{\omega \in \Omega} T_{\omega}$ be the orthogonal sum of an arbitrary family of completely hyperexpansive operators $T_{\omega} \in \boldsymbol{B}\left(\mathcal{H}_{\omega}\right)$.

(i) If $T$ is bounded, then $T$ is completely hyperexpansive and

$$
\begin{aligned}
F_{T}(\sigma) & =\bigoplus_{\omega \in \Omega} F_{T_{\omega}}(\sigma), \quad \sigma \in \mathcal{B}([0,1]), \\
\int_{[0,1]} \psi d F_{T} & =\bigoplus_{\omega \in \Omega} \int_{[0,1]} \psi d F_{T_{\omega}}, \quad \psi \in L^{1}\left(F_{T}\right),
\end{aligned}
$$

where $F_{T}$ and $F_{T_{\omega}}$ are operator measures associated with $T$ and $T_{\omega}$ respectively.

(ii) If every $T_{\omega}, \omega \in \Omega$, has completely hyperexpansive $k$-step full backward extension, then $T$ is bounded, it has completely hyperexpansive $k$-step full backward extension and (5.13) holds for $\psi=\varphi_{k}$.

Proof. (i) That $T$ is completely hyperexpansive follows directly from Definition 2.1. Applying (5.5) to $T_{\omega}$ and $n=1$, we conclude that

$$
\begin{aligned}
\left\|F_{T_{\omega}}(\sigma)\right\| & \leq\left\|F_{T_{\omega}}([0,1])\right\|=\left\|T_{\omega}^{*} T_{\omega}-I_{\mathcal{H}_{\omega}}\right\| \leq 1+\left\|T_{\omega}\right\|^{2} \\
& \leq 1+\|T\|^{2}, \quad \sigma \in \mathcal{B}([0,1]), \omega \in \Omega .
\end{aligned}
$$

This implies that for every $\sigma \in \mathcal{B}([0,1])$, the operator $F(\sigma):=\bigoplus_{\omega \in \Omega} F_{T_{\omega}}(\sigma)$ is bounded and $F$ is a positive-operator-valued Borel measure on $[0,1]$. Using (5.14), we see that $\int_{[0,1]} \psi d F=\bigoplus_{\omega \in \Omega} \int_{[0,1]} \psi d F_{T_{\omega}}$ for every bounded Borel function $\psi$ on $[0,1]$. Applying the last equality to the functions $\psi(x)=$ $1+x+\cdots+x^{n-1}$, we get

$$
T^{* n} T^{n}=I+\int_{[0,1]}\left(1+x+\cdots+x^{n-1}\right) F(d x), \quad n \geq 1 .
$$


In consequence, by Theorem 5.4, $F_{T}=F$, which proves (5.12). Take $\psi \in$ $L^{1}\left(F_{T}\right)$. It follows from (5.8) that $\psi \in L^{1}\left(F_{T_{\omega}}\right)$ and

$$
\left\|\int_{[0,1]} \psi d F_{T_{\omega}}\right\| \leq\left\|\int_{[0,1]}|\psi| d F_{T_{\omega}}\right\| \leq\left\|\int_{[0,1]}|\psi| d F_{T}\right\|, \quad \omega \in \Omega .
$$

This means that the operator $\bigoplus_{\omega \in \Omega} \int_{[0,1]} \psi d F_{T_{\omega}}$ is bounded. It is now a routine matter to show that $(5.13)$ is valid.

(ii) The operator $T$ is bounded because by (5.10), $\left\|T_{\omega}\right\| \leq \sqrt{(k+1) / k}$ for all $\omega \in \Omega$. Take $h=\oplus_{\omega \in \Omega} h_{\omega} \in \bigoplus_{\omega \in \Omega} \mathcal{H}_{\omega}$. By $(5.12),\left\langle F_{T}(\sigma) h, h\right\rangle=$ $\sum_{\omega \in \Omega}\left\langle F_{T_{\omega}}(\sigma) h_{\omega}, h_{\omega}\right\rangle$ for every $\sigma \in \mathcal{B}([0,1])$. Employing now the standard approximation procedure and Lebesgue's monotone convergence theorem for integrals and series, we get

$$
\int_{[0,1]} \psi(x)\left\langle F_{T}(d x) h, h\right\rangle=\sum_{\omega \in \Omega} \int_{[0,1]} \psi(x)\left\langle F_{T_{\omega}}(d x) h_{\omega}, h_{\omega}\right\rangle
$$

for every nonnegative Borel function $\psi$ on $[0,1]$. Suppose that $h$ is nonzero. Applying (5.6), (5.15) to $\psi=\varphi_{k}$ and part $1^{\circ}$ of Theorem 4.2, we obtain

$$
\begin{aligned}
\int_{[0,1]} \varphi_{k}(x) d \mu_{T, h}(x) & =\frac{1}{\|h\|^{2}} \int_{[0,1]} \varphi_{k}(x)\left\langle F_{T}(d x) h, h\right\rangle \\
& =\frac{1}{\|h\|^{2}} \sum_{\omega \in \Omega} \int_{[0,1]} \varphi_{k}(x)\left\langle F_{T_{\omega}}(d x) h_{\omega}, h_{\omega}\right\rangle \\
& =\frac{1}{\|h\|^{2}} \sum_{\substack{\omega \in \Omega \\
h_{\omega} \neq 0}}\left\|h_{\omega}\right\|^{2} \int_{[0,1]} \varphi_{k}(x) d \mu_{T_{\omega}, h_{\omega}}(x)<1 .
\end{aligned}
$$

According to Theorem 4.2 and Definition 5.1, $T$ has completely hyperexpansive $k$-step full backward extension. In view of the equivalence (i) $\Leftrightarrow$ (ii) of Theorem 5.6 and (5.13), we see that (5.13) holds for $\psi=\varphi_{k}$, which completes the proof.

6. Back to weighted shifts. We now return to the case of weighted shifts. We begin with a result which may be thought of as the complete hyperexpansive counterpart of [24, Corollary 4.4] (see also [17, Corollary 5.4]).

Proposition 6.1. Let $k \geq 1$ be an integer and $W$ be a completely hyperexpansive weighted shift. Then the following conditions are equivalent:

(i) W has completely hyperexpansive $k$-step full backward extension,

(ii) $W$ has a completely hyperexpansive $k$-step backward extension (or equivalently: $\left.e_{0} \in \mathcal{E}_{W, k}\right)$,

(iii) $\varphi_{k} \in L^{1}\left(F_{W}\right)$ and $\left\|\int_{[0,1]} \varphi_{k} d F_{W}\right\|<1$. 
Proof. The implication (i) $\Rightarrow$ (ii) is obvious.

(ii) $\Rightarrow$ (iii). Suppose that $e_{0} \in \mathcal{E}_{W, k}$. Let $\mu$ be the (scalar) measure associated with $W$. By (5.8) and Proposition 6.1 of [20], we have $L^{1}(\mu)=L^{1}\left(F_{W}\right)$ and

$$
\left\|\int_{[0,1]}|\psi| d F_{W}\right\|=\int_{[0,1]}|\psi| d \mu \quad \text { for every } \psi \in L^{1}(\mu)
$$

This and part $1^{\circ}$ of Theorem 4.2 imply (iii).

The implication (iii) $\Rightarrow(\mathrm{i})$ is a direct consequence of Theorem 5.6.

REMARK 6.2. Let $W$ be a completely hyperexpansive weighted shift with associated operator measure $F_{W}$. By [20, Proposition 6.1], $F_{W}$ is of the form

$$
F_{W}(\sigma) e_{n}=\mu_{n}(\sigma) e_{n}, \quad \sigma \in \mathcal{B}([0,1]), n \geq 0
$$

where

$$
\begin{aligned}
\mu_{0} & =\mu, \\
\mu_{n}(\sigma) & =\frac{\int_{\sigma} x^{n} d \mu(x)}{1+\int_{[0,1]}\left(1+x+\cdots+x^{n-1}\right) d \mu(x)}, \quad \sigma \in \mathcal{B}([0,1]), n \geq 1 .
\end{aligned}
$$

One can check that for every $\psi \in L^{1}(\mu)=L^{1}\left(F_{W}\right)$, the operator $\int_{[0,1]} \psi d F_{W}$ is diagonal (with respect to the orthonormal basis $\left\{e_{n}\right\}_{n=0}^{\infty}$ of $\ell^{2}$ ) with the diagonal $\left\{d_{n}\right\}_{n=0}^{\infty}$ given by

$$
d_{0}=\int_{[0,1]} \psi d \mu, \quad d_{n}=\frac{\int_{[0,1]} x^{n} \psi(x) d \mu(x)}{1+\int_{[0,1]}\left(1+x+\cdots+x^{n-1}\right) d \mu(x)}, \quad n \geq 1
$$

EXAMPle 6.3. Condition (iii) of Proposition 6.1 characterizes weighted shifts having completely hyperexpansive $k$-step full backward extensions. One might expect that this would be true for all completely hyperexpansive operators. However, this is not the case. Indeed, consider the sequence $\alpha_{n}=$ $n /(1+k n), n \geq 1$. Let $W^{(\alpha)}, \alpha \geq 0$, be as in Example 4.7. Since $k \alpha_{n}<1$ for all $n \geq 1$, we infer from Theorem 4.2 and Proposition 6.1 that each weighted shift $W^{\left(\alpha_{n}\right)}$ has completely hyperexpansive $k$-step full backward extension. In view of Proposition 5.8, the operator $T:=\bigoplus_{n=1}^{\infty} W^{\left(\alpha_{n}\right)}$ is completely hyperexpansive (in fact 2-isometric) and it has completely hyperexpansive $k$-step full backward extension. By $(6.1)$, we have

$$
\left\|\int_{[0,1]} \varphi_{k} d F_{W^{\left(\alpha_{n}\right)}}\right\|=k \alpha_{n}, \quad n \geq 1
$$

It follows from part (ii) of Proposition 5.8 and (6.4) that $\varphi_{k} \in L^{1}\left(F_{T}\right)$ and

$$
\left\|\int_{[0,1]} \varphi_{k} d F_{T}\right\|=\sup _{n \geq 1}\left\|\int_{[0,1]} \varphi_{k} d F_{W^{\left(\alpha_{n}\right)}}\right\|=\sup _{n \geq 1} k \alpha_{n}=1 .
$$


We now return to completely hyperexpansive $k$-step backward extension vectors in the context of weighted shifts.

Proposition 6.4. Let $k \geq 1$ be an integer and $W$ be a completely hyperexpansive weighted shift with associated measure $\mu$. Then (cf. Definition $5.1)$

(i) a nonzero vector $\left\{\eta_{n}\right\}_{n=0}^{\infty} \in \ell^{2}$ belongs to $\mathcal{E}_{W, k}$ if and only if

$\left|\eta_{0}\right|^{2} \int_{[0,1]} \varphi_{k} d \mu+\sum_{n=1}^{\infty}\left|\eta_{n}\right|^{2} \frac{\int_{[0,1]} x^{n} \varphi_{k}(x) d \mu(x)}{1+\int_{[0,1]}\left(1+x+\cdots+x^{n-1}\right) d \mu(x)}<\sum_{n=0}^{\infty}\left|\eta_{n}\right|^{2}$,

(ii) $\bigvee_{n \geq k}\left\{e_{n}\right\} \subseteq \mathcal{E}_{W, k}$.

Proof. (i) Using (5.6), (6.2), (6.3) and Theorem 4.2 (see also (5.15)), we conclude that a nonzero vector $h=\left\{\eta_{n}\right\}_{n=0}^{\infty} \in \ell^{2}$ belongs to $\mathcal{E}_{W, k}$ if and only if

$$
\begin{aligned}
1 & >\int_{[0,1]} \varphi_{k}(x) d \mu_{W, h}(x)=\frac{1}{\|h\|^{2}} \sum_{n=0}^{\infty}\left|\eta_{n}\right|^{2} \int_{[0,1]} \varphi_{k} d \mu_{n} \\
& =\frac{1}{\|h\|^{2}}\left(\left|\eta_{0}\right|^{2} \int_{[0,1]} \varphi_{k} d \mu+\sum_{n=1}^{\infty}\left|\eta_{n}\right|^{2} \frac{\int_{[0,1]} x^{n} \varphi_{k}(x) d \mu(x)}{1+\int_{[0,1]}\left(1+x+\cdots+x^{n-1}\right) d \mu(x)}\right) .
\end{aligned}
$$

(ii) By Proposition 5.2, $W^{k}\left(\ell^{2}\right)$ is a closed linear subspace of $\ell^{2}$. Hence we have $W^{k}\left(\ell^{2}\right)=\bigvee_{n \geq k}\left\{e_{n}\right\}$. Applying once more Proposition 5.2 completes the proof of (ii) (one can also deduce (ii) directly from (i)).

It is worth noting that part (i) of Proposition 6.4 implies the equivalence (i) $\Leftrightarrow$ (ii) of Proposition 6.1 as well as part $1^{\circ}$ of Theorem 4.2 (however, the latter has been essentially used in the proof of Proposition 6.4).

EXAMPLE 6.5. Let $k \geq 1$ be an integer. Consider the 2-isometric weighted shift $T:=W^{(1 / k)}$ with associated measure $\mu=(1 / k) \delta_{1}$ (cf. Example 4.7). It is easily seen that $T$ is unitarily equivalent to $W^{(1)} \mid \bigvee_{n=k-1}^{\infty}\left\{e_{n}\right\}$, where $W^{(1)}$ is the Dirichlet shift. Hence $T$ has no completely hyperexpansive $k$-step backward extension, though (for $k \geq 2$ ) it has a completely hyperexpansive $(k-1)$-step backward extension. We show that the set $\mathcal{E}_{T, k}$ is not a linear space and consequently it is not equal to $\bigvee_{n \geq k}\left\{e_{n}\right\}$. Notice that the counterpart of $\mathcal{E}_{T, 1}$ in the context of subnormal operators is equal to $\bigvee_{n \geq 1}\left\{e_{n}\right\}$ (cf. [17, Corollary 5.4]). By Proposition 6.4, a nonzero vector $h=\left\{\eta_{n}\right\}_{n=0}^{\infty} \in \ell^{2}$ belongs to $\mathcal{E}_{T, k}$ if and only if

$$
\sum_{n=1}^{\infty}\left|\eta_{n}\right|^{2} \frac{k}{k+n}<\sum_{n=1}^{\infty}\left|\eta_{n}\right|^{2}
$$

This means that $\mathcal{E}_{T, k}=\left\{\left\{\eta_{n}\right\}_{n=0}^{\infty} \in \ell^{2}: \exists m \geq 1 \quad \eta_{m} \neq 0\right\} \cup\{0\}$. 
EXAMPLE 6.6. Let $k, r \geq 1$ be integers and let $W$ be a completely hyperexpansive weighted shift with associated measure $\mu$. Suppose that $W$ has a completely hyperexpansive $(k+r)$-step backward extension. For $t \in[0, \infty)$, denote by $V_{t}$ the completely hyperexpansive $k$-step backward extension of $W$ associated with the measure $\zeta_{t}$ given by the right-hand side of (4.2). By Proposition 5.2 and Corollary 5.3, we identify $W$ (up to unitary equivalence) with $T_{k}:=\left.T\right|_{T^{k}(\mathcal{H})}$, where $\mathcal{H}=\ell^{2}$ and $T=V_{t}$. It follows from Remark 6.2 that $F_{V_{0}}(\{0\})=0$ and $F_{V_{t}}(\{0\}) \neq 0$ for $t>0$. By Proposition 6.1 and Corollary 5.3, $V_{0}$ has completely hyperexpansive $r$-step full backward extension. On the other hand, if $t>0$, then by Theorem 3.2, $V_{t}$ does not have any completely hyperexpansive backward extension, and consequently it does not have completely hyperexpansive full backward extension.

We will show in Example 6.10 below that similarity does not preserve the property of having completely hyperexpansive $k$-step full backward extension. Let us begin with a characterization of similarity of completely hyperexpansive weighted shifts associated with measures of tempered growth at 1, i.e. satisfying the condition (6.5) below which is closely related to power boundedness of completely hyperexpansive weighted shifts (cf. [20, Proposition 6.3]).

Proposition 6.7. Let $W_{1}$ and $W_{2}$ be completely hyperexpansive weighted shifts with associated measures $\mu_{1}$ and $\mu_{2}$, respectively. Assume that

$$
\int_{[0,1)} \frac{1}{1-x} d \mu_{i}(x)<\infty, \quad i=1,2 .
$$

Then the weighted shifts $W_{1}$ and $W_{2}$ are similar if and only if

$$
\text { either } \mu_{1}(\{1\})+\mu_{2}(\{1\})=0 \text { or } \mu_{1}(\{1\}) \cdot \mu_{2}(\{1\})>0 .
$$

Proof. Indeed, by [16, Problem 76] and (2.2), the weighted shifts $W_{1}$ and $W_{2}$ are similar if and only if there exist constants $c, d>0$ such that

$$
\begin{gathered}
c \leq \frac{\beta_{n+1}\left(W_{1}\right)}{\beta_{n+1}\left(W_{2}\right)}=\frac{1+(n+1) \mu_{1}(\{1\})+\int_{[0,1)} \frac{1-x^{n+1}}{1-x} d \mu_{1}(x)}{1+(n+1) \mu_{2}(\{1\})+\int_{[0,1)} \frac{1-x^{n+1}}{1-x} d \mu_{2}(x)} \leq d, \\
\quad n \geq 0 .
\end{gathered}
$$

Applying Lebesgue's monotone convergence theorem, we get

$$
\lim _{n \rightarrow \infty} \int_{[0,1)} \frac{1-x^{n+1}}{1-x} d \mu_{i}(x)=\int_{[0,1)} \frac{1}{1-x} d \mu_{i}(x), \quad i=1,2,
$$

which, together with (6.5) and (6.7), completes the proof.

Proposition 6.8. Let $W$ be a completely hyperexpansive weighted shift with associated measure $\mu$. Then 
(i) $W$ is similar to the isometric unilateral shift if and only if $W$ is power bounded, or equivalently

$$
\mu(\{1\})=0 \text { and } \int_{[0,1)} \frac{1}{1-x} d \mu(x)<\infty,
$$

(ii) $W$ is similar to the Dirichlet shift if and only if $\mu(\{1\})>0$.

Moreover, the isometric unilateral shift and the Dirichlet shift are not similar.

Proof. (i) If the weighted shift $W$ is power bounded, then by [20, Proposition 6.3] the condition (6.8) is valid. If (6.8) holds, then in view of Proposition 6.7 the weighted shift $W$ is similar to the isometric unilateral shift $W^{(0)}$ (because $W^{(0)}$ is associated with the zero measure). Finally, if $W$ and $W^{(0)}$ are similar, then $W$ is evidently power bounded.

(ii) If $\mu(\{1\})>0$, then by (2.2) we have

$$
\frac{1+(n+1) \mu(\{1\})}{1+(n+1)} \leq \frac{\beta_{n+1}(W)}{\beta_{n+1}\left(W^{(1)}\right)} \leq \frac{1+(n+1) \mu([0,1])}{1+(n+1)}, \quad n \geq 0
$$

where $W^{(1)}$ is the Dirichlet shift (cf. Example 4.7). Hence, by [16, Problem 76], the weighted shifts $W$ and $W^{(1)}$ are similar.

Suppose now that $\mu(\{1\})=0$. We define $\varepsilon_{n}=\int_{[0,1]} x^{n} d \mu(x)$ and $\sigma_{n}=$ $\left(\varepsilon_{0}+\cdots+\varepsilon_{n}\right) /(n+1)$ for $n \geq 0$. By Lebesgue's dominated convergence theorem, we have $\lim _{n \rightarrow \infty} \varepsilon_{n}=\mu(\{1\})=0$, and consequently $\lim _{n \rightarrow \infty} \sigma_{n}=0$. This in turn leads to

$$
\inf _{n \geq 0} \frac{\beta_{n+1}(W)}{\beta_{n+1}\left(W^{(1)}\right)}=\inf _{n \geq 0} \frac{\frac{1}{n+1}+\sigma_{n}}{\frac{n+2}{n+1}}=0,
$$

which in view of [16, Problem 76] implies that the weighted shifts $W$ and $W^{(1)}$ are not similar. This proves (ii).

Applying (i) to $W=W^{(1)}$ (or (ii) to $W=W^{(0)}$ ), we conclude that the operators $W^{(0)}$ and $W^{(1)}$ are not similar. This completes the proof.

We now indicate an example of a completely hyperexpansive weighted shift which is similar neither to the isometric unilateral shift nor to the Dirichlet shift.

EXAMPLE 6.9. Let $W$ be the completely hyperexpansive weighted shift associated with the Lebesgue measure on $[0,1]$. Since the sequence

$$
\frac{\beta_{n+1}(W)}{\beta_{n+1}\left(W^{(0)}\right)}=1+\left(\frac{1}{1}+\cdots+\frac{1}{n+1}\right), \quad n \geq 0,
$$

is unbounded, the weighted shift $W$ is not similar to the isometric unilateral 
shift $W^{(0)}$. On the other hand, since

$$
\begin{aligned}
\frac{\beta_{n+1}(W)}{\beta_{n+1}\left(W^{(1)}\right)} & =\frac{2+\left(\frac{1}{2}+\cdots+\frac{1}{n+1}\right)}{1+(n+1)} \\
& \leq \frac{2+\int_{1}^{n+1} \frac{1}{x} d x}{n+1}=\frac{2}{n+1}+\frac{\log (n+1)}{\mathrm{e}^{\log (n+1)}}, \quad n \geq 1,
\end{aligned}
$$

we get $\inf _{n \geq 1} \beta_{n+1}(W) / \beta_{n+1}\left(W^{(1)}\right)=0$. Hence the weighted shift $W$ is not similar to the Dirichlet shift $W^{(1)}$.

EXAMPLE 6.10. Since the conditions (6.5) and (6.6) are not particularly restrictive, one cannot expect that the property of having completely hyperexpansive $k$-step full backward extension is preserved by similarity. For instance, if $\mu_{1}$ is such that $0<\alpha:=\int_{[0,1]} \varphi_{k} d \mu_{1}<1$ and $\mu_{2}=(1 / \alpha) \mu_{1}$, then $W_{1}$ and $W_{2}$ are similar $\left({ }^{7}\right), W_{1}$ has completely hyperexpansive $k$-step full backward extension, while $W_{2}$ does not (cf. Theorem 4.2 and Proposition 6.1). In particular, the measures $\mu_{1}=\frac{1}{k+1} \delta_{1}$ and $\mu_{2}=\frac{1}{k} \delta_{1}$ have the feature we want.

We conclude this section with a version of Proposition 6.1 for operatorvalued (unilateral) weighted shifts $T$ with invertible weights $\left\{T_{n}\right\}_{n=0}^{\infty}$ whose products $T_{n} \cdots T_{0}, n \geq 0$, are positive. We follow the notation of [22]. The reader should be aware of the fact that the operator measure $G$ appearing in [22] is the same as our $F_{T}$.

Proposition 6.11. If $T, F$ and $G$ are as in Section 4 of [22] and $k \geq 1$ is an integer, then the following conditions are equivalent:

(i) T has completely hyperexpansive $k$-step full backward extension,

(ii) $\varphi_{k} \in L^{1}(F)$ and $\int_{[0,1]} \varphi_{k} d F<I_{\mathcal{H}}$,

(iii) $\varphi_{k} \in L^{1}(F),\left\|\int_{[0,1]} \varphi_{k} d F\right\| \leq 1$ and 1 is not an eigenvalue of the operator $\int_{[0,1]} \varphi_{k} d F$.

Sketch of the proof. (ii) $\Rightarrow$ (i). In view of [22, Theorem 4.2], $\varphi_{k} \in L^{1}(F)=$ $L^{1}(G)$. Since $\int_{[0,1]} \varphi_{k} d F<I_{\mathcal{H}}$, we have (see [22, p. 414, line 10 from above])

$$
\begin{aligned}
& \int_{[0,1]} \varphi_{k}(x)\left\langle F_{i}(d x) h, h\right\rangle=\int_{[0,1]} x^{i} \varphi_{k}(x)\left\langle F(d x) \Omega_{F, i}^{-1} h, \Omega_{F, i}^{-1} h\right\rangle \\
& \leq\left\langle\left(\int_{[0,1]} \varphi_{k} d F\right) \Omega_{F, i}^{-1} h, \Omega_{F, i}^{-1} h\right\rangle<\left\langle\Omega_{F, i}^{-1} h, \Omega_{F, i}^{-1} h\right\rangle \leq\langle h, h\rangle
\end{aligned}
$$

$\left({ }^{7}\right)$ This can be justified simply by computing $\lim _{n \rightarrow \infty} \beta_{n+1}\left(W_{1}\right) / \beta_{n+1}\left(W_{2}\right)$. 
for $i \geq 0$ and $h \in \mathcal{H} \backslash\{0\}$. This and (5.7) imply

$$
\varphi_{k} \in L^{1}\left(F_{i}\right) \text { and } \int_{[0,1]} \varphi_{k} d F_{i}<I_{\mathcal{H}} \quad \text { for all } i \geq 0 .
$$

Since the operator measure $G$ equals the orthogonal sum of the positiveoperator-valued Borel measures $F_{i},(6.9)$ enables us to show that

$$
\int_{[0,1]} \varphi_{k} d G=\bigoplus_{i=0}^{\infty} \int_{[0,1]} \varphi_{k} d F_{i}
$$

Employing Theorem 5.6, (6.9) and (6.10), we see that (i) holds.

(i) $\Rightarrow$ (ii). By Theorem 5.6, $\varphi_{k} \in L^{1}(G)$ and $\int_{[0,1]} \varphi_{k} d G<I_{\ell^{2}(\mathcal{H})}$. Since $F(\sigma)=F_{0}(\sigma) \subseteq G(\sigma)$ for $\sigma \in \mathcal{B}([0,1])$, we conclude that (ii) holds.

The equivalence (ii) $\Leftrightarrow($ iii) follows from (5.11).

7. 2-hyperexpansive $k$-step backward extensions. In this concluding section we briefly discuss 2-hyperexpansive backward extensions leaving the question of $m$-hyperexpansive backward extensions aside. One can easily adapt Definitions 3.1, 4.1 and 5.1 to the context of 2-hyperexpansive operators, simply by replacing "completely hyperexpansive" by "2-hyperexpansive". For the reader's convenience, we collect indispensable facts concerning 2hyperexpansive weighted shifts. By Proposition 6.2 of [23], the weight sequence $\left\{\lambda_{n}\right\}_{n=0}^{\infty}$ of a 2-hyperexpansive weighted shift $W$ satisfies the following conditions:

$4^{\mathrm{o}} \quad \lambda_{n} \geq 1$ for all $n \geq 0$,

$5^{\circ} \quad \lambda_{n+1} \leq \lambda_{n}$ for all $n \geq 0$,

$6^{\circ} \quad \lambda_{n} \leq \sigma_{n}\left(\lambda_{0}\right):=\sqrt{\frac{1+(n+1)\left(\lambda_{0}^{2}-1\right)}{1+n\left(\lambda_{0}^{2}-1\right)}}$ for all $n \geq 0$,

$7^{\circ}$ if $\lambda_{0}=1$, then $\lambda_{n}=1$ for all $n \geq 0$.

Proposition 7.1. Let $W$ be a 2-hyperexpansive weighted shift with weight sequence $\left\{\lambda_{n}\right\}_{n=0}^{\infty}$ and let $k \geq 1$ be an integer. Then the following conditions are equivalent:

(i) $W$ has a 2-hyperexpansive $k$-step backward extension,

(ii) $\lambda_{0}<\sqrt{(k+1) / k}$.

Moreover, $W$ has a 2-hyperexpansive $k$-step backward extension for all $k \geq 1$ if and only if $W$ is the isometric unilateral shift.

Proof. (i) $\Rightarrow$ (ii). Suppose that there are positive scalars $\lambda_{-k}, \ldots, \lambda_{-1}$ such that the weighted shift $V$ with weight sequence $\left\{\lambda_{n-k}\right\}_{n=0}^{\infty}$ is 2-hyper- 
expansive. Set $\alpha=\lambda_{-k}^{2}-1$. Applying $4^{\circ}$ and $6^{\circ}$ to $V$, we get $\alpha \geq 0$ and

$$
\lambda_{0} \leq \sigma_{k}\left(\lambda_{-k}\right)=\sqrt{\frac{1+(k+1) \alpha}{1+k \alpha}}=\sqrt{1+\frac{\alpha}{1+k \alpha}}<\sqrt{\frac{k+1}{k}} .
$$

(ii) $\Rightarrow$ (i). By $4^{\mathrm{o}}, \alpha:=\lambda_{0}^{2}-1 \geq 0$. Let $W^{(\alpha)}$ be as in Example 4.7. Since (ii) is equivalent to $k \alpha<1$, we see that $W^{(\alpha)}$ has a completely hyperexpansive (and consequently 2-hyperexpansive) $k$-step backward extension $W^{\prime}$ with weight sequence

$$
\lambda_{-k}, \ldots, \lambda_{-1}, \lambda_{0}=\sqrt{1+\alpha}, \sqrt{\frac{1+2 \alpha}{1+\alpha}}, \ldots, \sqrt{\frac{1+(n+1) \alpha}{1+n \alpha}}, \ldots
$$

Using Lemma 6.1(i) of [23], one can check that the weighted shift $V$ with weight sequence $\lambda_{-k}, \ldots, \lambda_{-1}, \lambda_{0}, \lambda_{1}, \ldots, \lambda_{n}, \ldots$ is a 2 -hyperexpansive $k$-step backward extension of $W$.

The remaining part of the conclusion can be deduced from $4^{\circ}, 7^{\circ}$ and the implication (i) $\Rightarrow$ (ii). This completes the proof.

Corollary 7.2. Let $k \geq 1$ be an integer. A 2-hyperexpansive operator $T \in \boldsymbol{B}(\mathcal{H})$ has 2-hyperexpansive $k$-step full backward extension if and only if (5.10) holds. A 2-hyperexpansive weighted shift $W$ with weight sequence $\left\{\lambda_{n}\right\}_{n=0}^{\infty}$ has 2-hyperexpansive $k$-step full backward extension if and only if $\lambda_{0}<\sqrt{(k+1) / k}$.

Proof. The case of $T \in \boldsymbol{B}(\mathcal{H})$ can be handled with the help of Propositions 2.4 and 7.1. By $5^{\mathrm{o}}, W^{*} W<\frac{k+1}{k} I_{\ell^{2}}$ if and only if $\lambda_{0}<\sqrt{(k+1) / k}$, which completes the proof.

REMARK 7.3. It is worth noting that in view of $5^{\circ}$ and Corollary 5.7 a 2isometric weighted shift $W$ with weight sequence $\left\{\lambda_{n}\right\}_{n=0}^{\infty}$ has completely hyperexpansive $k$-step full backward extension if and only if $\lambda_{0}<\sqrt{(k+1) / k}$ (this can also be deduced from Theorem 4.2 and Proposition 6.1). If a 2isometric operator $T \in \boldsymbol{B}(\mathcal{H})$ has completely hyperexpansive $k$-step full backward extension, then by Proposition 2.4 and Example 4.7, $T$ has 2isometric $k$-step full backward extension. This and Corollaries 5.7 and 7.2 imply that a 2-isometric operator $T \in \boldsymbol{B}(\mathcal{H})$ has 2-hyperexpansive $k$-step full backward extension if and only if it has 2-isometric $k$-step full backward extension.

Remark 7.4. It follows from Propositions 2.4 and 7.1 that if $T \in \boldsymbol{B}(\mathcal{H})$ is a 2-hyperexpansive operator, then a nonzero vector $h \in \mathcal{H}$ is a 2-hyperexpansive $k$-step backward extension vector for $T$ if and only if $\|T h\|<$ $\sqrt{(k+1) / k}\|h\|$. If $T \in \boldsymbol{B}(\mathcal{H})$ is a 2-isometry, then in view of the discussion in Remark 7.3 for every nonzero vector $h \in \mathcal{H}$ the following conditions are equivalent: 
(a) $h$ is a 2-hyperexpansive $k$-step backward extension vector for $T$,

(b) $h$ is a completely hyperexpansive $k$-step backward extension vector for $T$,

(c) $h$ is a 2-isometric $k$-step backward extension vector for $T$.

This and Example 6.5 show that the set of all 2-hyperexpansive $k$-step backward extension vectors for $T=W^{(1 / k)}$ together with the zero vector is not a linear space.

Acknowledgements. A substantial part of this paper was written while the first and third authors visited Kyungpook National University during the spring of 2004; they wish to thank the faculty and administration of this unit for their warm hospitality.

\section{References}

[1] J. Agler, Hypercontractions and subnormality, J. Operator Theory 13 (1985), 203217.

[2] —, A disconjugacy theorem for Toeplitz operators, Amer. J. Math. 112 (1990), 1-14.

[3] J. Agler and M. Stankus, m-isometric transformations of Hilbert space. I, Integral Equations Operator Theory 21 (1995), 383-429.

[4] -, - m-isometric transformations of Hilbert space. II, ibid. 23 (1995), 1-48.

[5] - - - m-isometric transformations of Hilbert space. III, ibid. 24 (1996), 379-421.

[6] A. Aleman, The multiplication operators on Hilbert spaces of analytic functions, Habilitationsschrift, Fernuversität Hagen, 1993.

[7] A. Athavale, On completely hyperexpansive operators, Proc. Amer. Math. Soc. 124 (1996), 3745-3752.

[8] -, The complete hyperexpansivity analog of the Embry conditions, Studia Math. 154 (2003), 233-242.

[9] A. Athavale and A. Ranjekar, Bernstein functions, complete hyperexpansivity and subnormality. I, Integral Equations Operator Theory 43 (2002), 253-263.

[10] - - - Bernstein functions, complete hyperexpansivity and subnormality. II, ibid. 44 (2002), 1-9.

[11] A. Athavale and V. M. Sholapurkar, Completely hyperexpansive operator tuples, Positivity 3 (1999), 245-253.

[12] R. E. Curto, Quadratically hyponormal weighted shifts, Integral Equations Operator Theory 13 (1990), 49-66.

[13] R. E. Curto and W. Y. Lee, $k$-hyponormality of finite rank perturbations of unilateral weighted shifts, Trans. Amer. Math. Soc. 357 (2005), 4719-4737.

[14] M. Embry, A generalization of the Halmos-Bram criterion for subnormality, Acta Sci. Math. (Szeged) 35 (1973), 61-64.

[15] T. Furuta, On the class of paranormal operators, Proc. Japan. Acad. 43 (1967), 594-598.

[16] P. R. Halmos, A Hilbert Space Problem Book, Van Nostrand, Princeton, NJ, 1967.

[17] T. Hoover, I. Jung and A. Lambert, Moment sequences and backward extensions of subnormal weighted shifts, J. Austral. Math. Soc. 73 (2002), 27-36.

[18] V. I. Istrățescu, On some hyponormal operators, Pacific J. Math. 22 (1967), 413-417.

[19] T. Ito and T. K. Wong, Subnormality and quasinormality of Toeplitz operators, Proc. Amer. Math. Soc. 34 (1972), 157-164. 
[20] Z. J. Jabłoński, Complete hyperexpansivity, subnormality and inverted boundedness conditions, Integral Equations Operator Theory 44 (2002), 316-336.

[21] - Hyperexpansive composition operators, Math. Proc. Cambridge Philos. Soc. 135 (2003), 513-526.

[22] - Hyperexpansive operator valued unilateral weighted shifts, Glasgow Math. J. 46 (2004), 405-416.

[23] Z. J. Jabłoński and J. Stochel, Unbounded 2-hyperexpansive operators, Proc. Edinburgh Math. Soc. 44 (2001), 613-629.

[24] I. Jung, A. Lambert and J. Stochel, Backward extensions of subnormal operators, Proc. Amer. Math. Soc. 132 (2004), 2291-2302.

[25] A. Lambert, Subnormality and weighted shifts, J. London Math. Soc. 14 (1976), $476-480$.

[26] S. McCullough, Sub-Brownian operators, J. Operator Theory 22 (1989), 291-305.

[27] S. Richter, Invariant subspaces of the Dirichlet shift, J. Reine Angew. Math. 386 (1988), 205-220.

[28] - A representation theorem for cyclic analytic two-isometries, Trans. Amer. Math. Soc. 328 (1991), 325-349.

[29] S. Shimorin, Wold-type decompositions and wandering subspaces for operators close to isometries, J. Reine Angew. Math. 51 (2001), 147-189.

[30] V. M. Sholapurkar and A. Athavale, Completely and alternatingly hyperexpansive operators, J. Operator Theory 43 (2000), 43-68.

[31] J. Stochel, Decomposition and disintegration of positive definite kernels on convex *-semigroups, Ann. Polon. Math. 56 (1992), 243-294.

Institute of Mathematics

Jagiellonian University

Reymonta 4

30-059 Kraków, Poland

E-mail: jablonsk@im.uj.edu.pl stochel@im.uj.edu.pl
Department of Mathematics

College of Natural Sciences Kyungpook National University Daegu 702-701 Korea E-mail: ibjung@knu.ac.kr 